\title{
Dynamic infrared thermography (DIRT) in Deep Inferior Epigastric Perforator (DIEP) flap breast reconstruction: standardization of the measurement set-up
}

\author{
Filip E. F. Thiessen ${ }^{1,2}$, Thierry Tondu ${ }^{1,2}$, Nicolas Vermeersch ${ }^{1}$, Ben Cloostermans ${ }^{3}$, Ralv Lundahl ${ }^{3}$, \\ Bart Ribbens ${ }^{3}$, Lawek Berzenji ${ }^{4}$, Veronique Verhoeven ${ }^{5}$, Guy Hubens ${ }^{6}$, Gunther Steenackers ${ }^{3}$, \\ Wiebren A. A. Tjalma ${ }^{7}$
}

${ }^{1}$ Department of Plastic, Reconstructive and Aesthetic Surgery, Multidisciplinary Breast Clinic, Antwerp University Hospital, University of Antwerp, Antwerp, Belgium; ${ }^{2}$ Department of Plastic, Reconstructive and Aesthetic Surgery, Ziekenhuis Netwerk Antwerpen, Antwerp, Belgium; ${ }^{3}$ Op3Mech Research Group, University of Antwerp, Antwerp, Belgium; ${ }^{4}$ Department of Thoracic and Vascular Surgery, Antwerp University Hospital, University of Antwerp, Antwerp, Belgium; ${ }^{5}$ Department of Primary and Interdisciplinary Care (ELIZA), University of Antwerp, Antwerp, Belgium; ${ }^{6}$ Department of Abdominal, Paediatric and Reconstructive Surgery, ${ }^{7}$ Gynaecological Oncology Unit, Department of Obstetrics and Gynaecology, Multidisciplinary Breast Clinic, Antwerp University Hospital, University of Antwerp, Antwerp, Belgium

Correspondence to: Filip E. F. Thiessen, MD. Department of Plastic, Reconstructive and Aesthetic Surgery, Multidisciplinary Breast Clinic, Antwerp University Hospital, University of Antwerp, Wilrijkstraat 10, 2650 Edegem, Antwerp, Belgium. Email: filip.thiessen@clinic12b.be.

\begin{abstract}
Breast reconstruction with an autologous free Deep Inferior Epigastric Perforator (DIEP) flap is one of the preferred options following mastectomy. A challenging step in this procedure is the selection of a suitable perforator that provides sufficient blood supply for the flap. The current golden standard for perforator mapping is computed tomography angiography (CTA). However, this is a relatively expensive imaging modality that requires intravenous contrast injection and exposes patients to ionizing radiation. More recently, dynamic infrared thermography (DIRT) has been proposed as an alternative imaging modality for perforator identification. DIRT appears to be an ideal alternative technique not only for the identification of the dominant perforators, but also for the mapping of the individual influence of each perforator on the flap perfusion. Multiple studies have been performed with the use of DIRT, unfortunately without standardisation of the measurement set-up. In this technical note we propose a standardised and reproducible measurement set-up for the use of DIRT during breast reconstructions with a free DIEP flap. This set-up can be used pre-, intra- and postoperatively. A standardised measurement set-up will improve the quality of measured data and ensure reproducibility.
\end{abstract}

Keywords: Breast reconstruction; Deep Inferior Epigastric Perforator flap (DIEP flap); dynamic infrared thermography (DIRT); medical thermography; perforator imaging

Submitted Aug 29, 2019. Accepted for publication Nov 29, 2019.

doi: 10.21037 /gs.2019.12.09

View this article at: http://dx.doi.org/10.21037/gs.2019.12.09

\section{Introduction}

Breast reconstruction with a Deep Inferior Epigastric Perforator (DIEP) flap is a common option for reconstruction after mastectomy. In breast reconstructions with DIEP flaps, the skin and subcutaneous tissue from the patient's lower abdomen are used as a free flap to reconstruct the breast. The flap receives its blood supply from the deep inferior epigastric artery and one or two concomitant veins through a perforator. Following dissection of the flap, the flap is anastomosed to the internal mammary artery and vein for an optimal blood supply (1-3).

One of the key elements in DIEP flap surgery is the selection of the best perforators. Optimal perforator selection reduces operation times, complication rates 
and ensures an overall better result. Current techniques to locate perforator vessels include handheld Doppler, colour Doppler ultrasound (CDU), magnetic resonance angiography (MRA), computer tomographic angiography (CTA), and dynamic infrared thermography (DIRT) $(4,5)$. The current golden standard for perforator selection is CTA on which the location and hemodynamic properties of the flap can be assessed. CTA is frequently used because it is non-invasive and has a high spatial resolution with visualisation of the intramuscular course of the vessels. However, this technique has disadvantages, such as the use of intravenous (IV) contrast agents and ionizing radiation, high purchasing costs, a lack of perioperative usability, and a lack of physiological information on flow characteristics of perforators (4).

An alternative technique that has gained popularity in recent years is DIRT. DIRT is less invasive than CTA because it does not use radiation nor contrast agents. It is based on measurements of heat emission by tissues and skin temperature with the use of infrared (IR)-cameras. Data obtained with DIRT can be used to generate colorcoded maps that strongly correlate to the perfusion of the skin. DIRT is generally used as a dynamic investigation technique, meaning that the skin must undergo a thermal cold challenge. After this cold challenge, DIRT measures the rate and patterns of rewarming. With this method, clinicians are able to identify the most dominant perforators and their perfusion area $(6,7)$.

Earlier studies have shown that DIRT can be a valuable addition during all phases of breast reconstructions with DIEP flaps. DIRT is a quick imaging technique that is available pre-, peri-, and post-operatively (7-14). DIRT is a valuable alternative to clinical examination to evaluate at any stage during surgery the perfusion of the flap (15). DIRT can also be an interesting alternative to the use of indocyanine green to evaluate the microcirculation and perfusion of the flap preoperatively. DIRT is less invasive than the use of indocyanine green because there is no need for contrast agents. Moreover the potential allergic reactions to ICG should be taken into consideration (16). Furthermore, DIRT is easy to interpret and has a low purchasing cost. On the other hand, DIRT only provides information on the physiology of the perforator and not on the morphology. This means that the surgeon must have a thorough knowledge of the vascular anatomy in order to interpret the results (6).

When applying DIRT in a clinical setting, there a lot of factors to take into account. The choice of cameras, software, and cooling methods are crucial for successful measurements. In medical literature the description of the measurement set-up for DIRT is very diverse (14). Only a standardised and reproducible measurement setup will improve the quality of the measured data and will make comparisons between subjects or studies possible. The goal of present technical note is to describe a new and standardized measurement method for the use of DIRT in the pre-, peri- and post-operative setting. The setup proposed in this study is based on a combination of several strategies found in earlier studies $(7-11,13,17)$.

\section{Material and methods}

\section{Cooling}

The introduction of a cold or heat challenge is recommended to create an even starting situation for the whole area of interest $(7,13,18)$. Locating the perforators is easier with a cold challenge because the appearing hotspots are clearer against an even background. Cooling ensures that only the areas which are best supplied with blood will become noticeable hotspots in the minutes after removal of the excitation (19). The reduction of the so called "fake hotspots" is critical for the accuracy of this method. Fake hotspots are small areas where superficial blood vessels give the impression of an underlying perforator. The implemented cooling method to achieve the introduction of the cold challenge is a sterile bag filled with water and ice. The water inside the bag has a temperature of 3 to $5{ }^{\circ} \mathrm{C}$. The bag is positioned on the abdomen for a certain cooling time. It has to cover the entire area of interest and follow the contour of the abdomen to ensure an even cooling of the area. Creases in the plastic bag can cause uneven cooling of the area of interest and may lead to false appearing hotspots after removal of the sterile bag (Figure 1).

This technique is the result of a comparison between different cooling methods:

First cooling with sterile compresses soaked in saline at 5 degrees was tested. This method was not ideal due to the interference of the water left behind on the surface at the beginning of the IR-measurement. The cooling was not even, nor was the amount of extracted heat from the abdomen sufficient.

Secondly, an aluminium plate wrapped in a sterile drape was pressed against the abdomen (7). This technique has been described, but was found unfit because of uneven cooling (Figure 1B). The thermal image showed warmer 

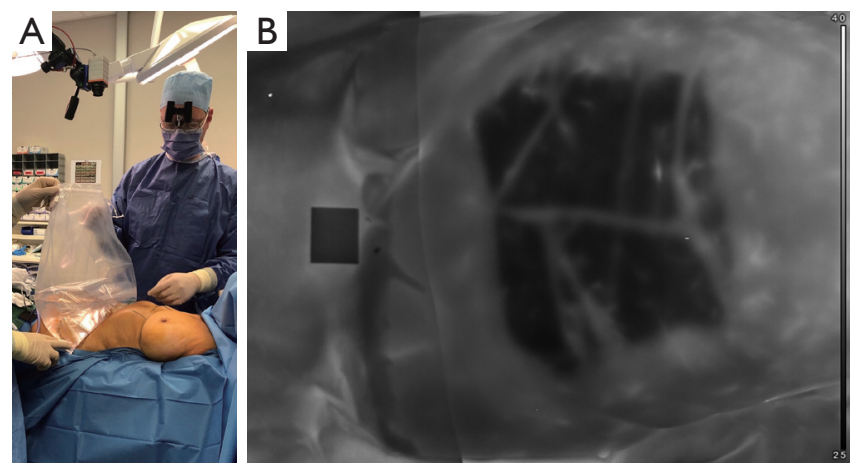

Figure 1 Different cooling methods. (A) Cooling with filled sterile bag; (B) image after cooling with aluminium plate.

lines across the abdomen where the creases had been. This made the image distorted and therefore not comparable.

Cooling with alcohol was tested (20). Alcohol extracts heat from the skin to evaporate and cools the skin. The evaporation happened too quickly and drained too little heat. No even cooling was obtained due to unevenly dispersed alcohol on the abdomen and fast evaporation time.

The parameters to hold into account when optimizing the cooling method are the cooling capacity, the contact surface, and the cooling time. The equation below shows the relation between these parameters.

$\mathrm{Q}=\mathrm{m} \cdot \mathrm{Cp} \cdot \Delta \mathrm{T} / \mathrm{t}$

where:

$\mathrm{Q}=$ Cooling capacity $(\mathrm{kW})$

$\mathrm{m}=$ mass $(\mathrm{kg})$

$\mathrm{Cp}=$ specific heat capacity of cooling liquid $(\mathrm{kJ} / \mathrm{kgK})$

$\Delta \mathrm{T}=$ temperature change $(\mathrm{K})$

$\mathrm{t}=$ cooling time $(\mathrm{s})$

The equation shows an inverse relation between cooling time and temperature change. To obtain an optimal cooling time while preventing tissue damage due to low temperatures, a balance between these parameters needs to be found.

In this set-up, a water temperature of $5{ }^{\circ} \mathrm{C}$ and a cooling time of 3 minutes are used to provide a sufficient cooling capacity without damaging tissues or creating a long cooling time.

\section{Infrared camera}

For the measurements, a thermal imaging camera is used. An uncooled, long wavelength microbolometer camera is used because of its compact size, high image resolution, and precision at relatively low temperature-measurements. In our setting we use the Xenics Gobi 640. The Gobi 640-series has a high sensitivity for small signals due to a low noise detector. A frame rate of $50 \mathrm{~Hz}$ is be used to see the emerging hotspots. We compared the quality of the images with the Xenics Gobi 640 to the FLIR X6800SC, with appropriate filters in order to match the wavelength range of the Xenics Gobi 640. The Flir IR camera has a quantum detector which means they have to be cooled to approximately $-196^{\circ} \mathrm{C}$. This makes this type of camera large and therefore less practical in an operating room, on top of that the quality of the results was similar. The long wave IR-cameras are less expensive compared to the medium wave cameras and are often used in technical and medical domains (21). For the dynamic measurements, the data consist of video files of 5 minutes. The recording starts at the moment the cooling (or vascular clamp) is removed from the area of interest.

\section{Tripod}

The IR-camera is positioned above the patient. The optimal position for the IR-camera is perpendicular to the abdominal area. In this position the camera can capture the clearest view of the flap (Figure 2). The fixed position of the camera will allow automated image analysis. Furthermore, the exact distance of the camera with respect to the measured object can be inserted as input for the software in order to measure accurate distances on the thermal image.

In order to obtain this perpendicular position above the abdomen of the patient, a tripod with an arm length of $250 \mathrm{~cm}$ including a counterweight is used. The tripod is mounted on wheels to enable manoeuvring. The tripod can be placed at the foot end of the operation table to prevent interference with the operating surgeon (Figure 3). The tripod is mounted on wheels to enable manoeuvring during surgery. To ensure every measurement has the exact same camera position relative to the operation table or bed, markings are drawn on the tripod and the ground. In our set-up, the Manfrotto Black Light Boom 025B tripod is used.

\section{Software and data transmission}

Importing and editing camera data was performed using Xeneth v2.6.0 (Xenics, Leuven). This software shows the taken images in real-time and allows the operator to adjust the scaling. Raw data can also be imported into Matlab 

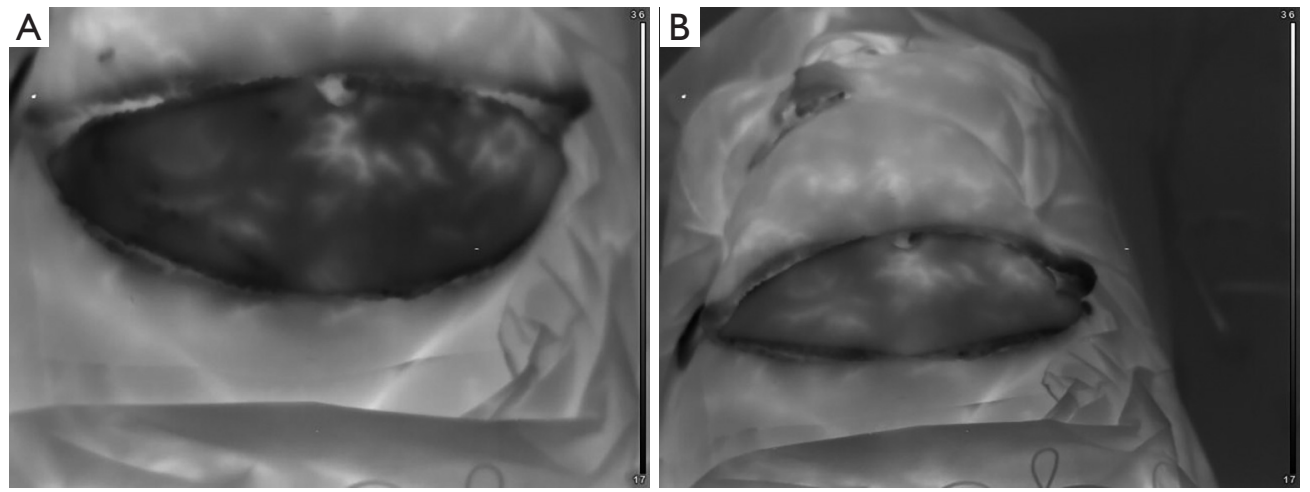

Figure 2 Camera positioned in different angles. (A) Perpendicular position of the camera; (B) camera at unknown angle.

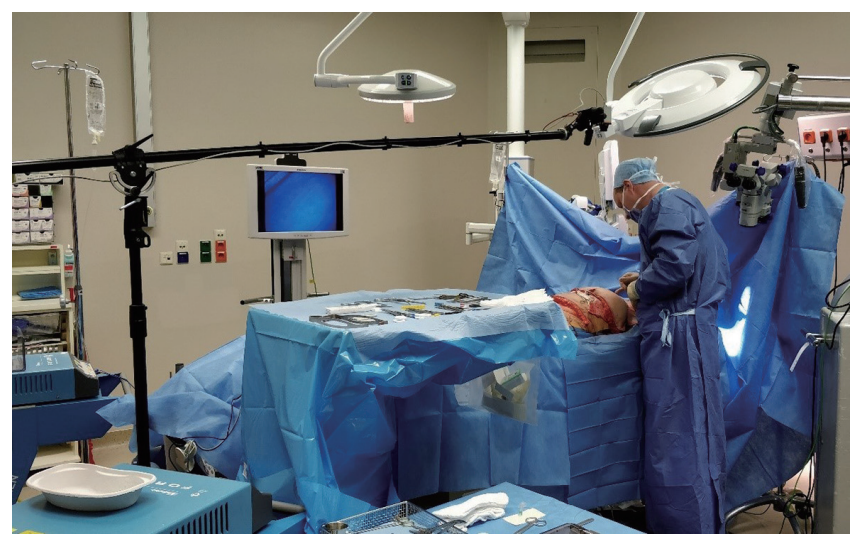

Figure 3 Position of the tripod and the camera in the operating room (not disturbing the surgeon during surgery).

(MathWorks, Natick, MA, USA) for further post processing.

The power supply of the thermal imaging camera and the data transfer is realized over one Power over Ethernet (PoE) cable.

\section{Measurement strategy}

The standardized measurement strategy makes the technique reproducible and ensures that different test subjects are comparable. A breast reconstruction with a DIEP flap can be divided into a preoperative, perioperative, and postoperative section.

\section{Preoperative}

A static image is made of the abdomen without cooling, as a control image. Subsequently, the cold challenge is performed and recording of the measurements is started. The cold challenge induces the appearance of hotspots which identify the dominant perforators $(9,10)$.

\section{Perioperative}

The perioperative measurements are taken after dissection of the perforators $(22,23)$. The first measurement is a recording after the cold challenge with all the dissected perforators open/unclamped. A comparison is made between the hotspots and the actual perforators to see whether they correlate (Figure 4). The goal of the second intraoperative measurement is to map the specific influence of each perforator on the perfusion of the flap. A microvascular clamp is used to clamp the perforator temporarily until the flap shows no heated areas. Subsequently, one of the clamps is removed and the recording is started. This provides a good visualization of the influence of the perforator on flap perfusion (Figure 5). This procedure is repeated according to the number of perforators that are dissected.

The last measurement takes place after the flap is anastomosed. Recording starts immediately after opening the venous and arterial anastomosis. The flap undergoes a cold challenge due to the non-perfusion during flap ischemia. An extra cold challenge can be performed after the anastomosis when needed. The last measurement determines the influence of the anastomosis on the flap perfusion. These data can be used to identify, and discard, less vascularized parts of the flap (Figure 6).

\section{Postoperative}

In the postoperative period, DIRT can potentially detect 

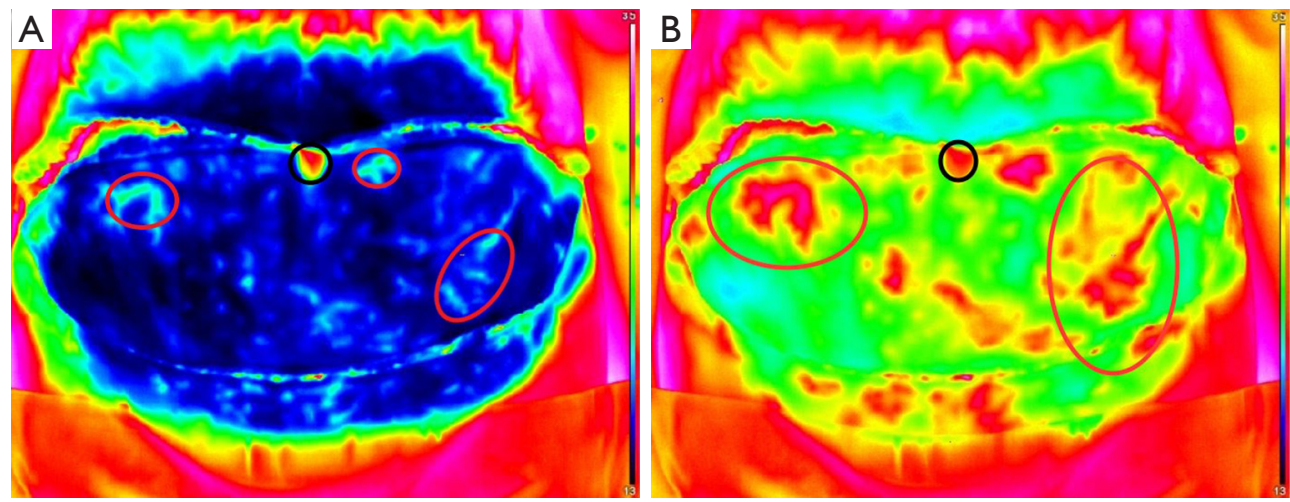

Figure 4 Intraoperative measurement with cooling. (A) Cooled abdominal area with the hotspots encircled; (B) 4 minutes after removal of cold challenge. Hotspots encircled.
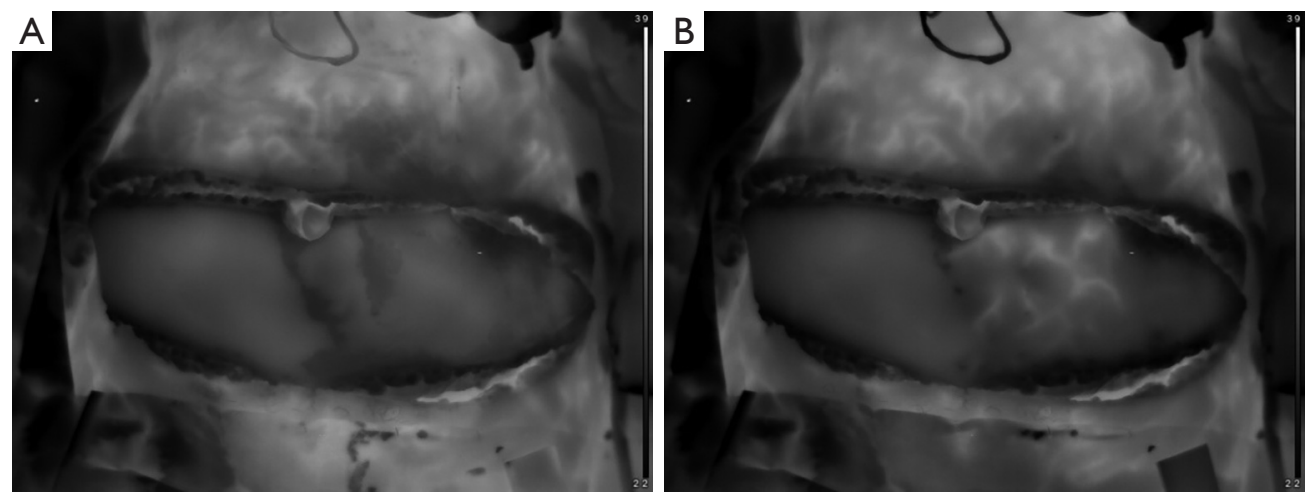

Figure 5 Intraoperative measurement with clamps. (A) Initial state (immediate after clamp removal); (B) steady state (4 minutes after clamp removal).

early (partial) necrosis. After 1-2 postoperative days, 2 measurements will be taken: 1 static image of the reconstructed breast and 1 dynamic image after a cold challenge. The same measurement set-up as during surgery can be used on the ward.

\section{Discussion}

Dynamic thermography in breast reconstructions with DIEP flaps has shown to be a valuable addition in the pre-, peri- and postoperative period. DIRT is able to measure the rate and patterns of rewarming after cooling, which allows clinicians to identify the dominant perforators and the perfusion-area $(6,7,14)$.

There is a great variety in measurement set-ups in literature which makes comparisons between the different techniques difficult. The introduction of the cold challenge is very variable in literature. de Weerd $e t$ al. introduce a cold challenge by blowing air over the abdomen for 2 minutes using a desktop fan. Afterwards, a recording is made with an IR camera for 3 minutes $(6,7,10,24,25)$. The difficulty with using a cold air stream is to obtain an even and homogenous cooling of the abdomen. Furthermore, the cooling capacity of cold air is lower than that of cold water. This can potentially cause bigger hotspots and complicate pinpointing the exact location of perforators. In addition to this, cooling with a desktop fan is impossible in the operation room. Another technique is the use of a metal plate to introduce a cold challenge perioperatively (7). This metal plate only allows cooling of a small area and does not follow the curve of the abdomen. Water-packs have been used to introduce a cold challenge (13). In this study, a sterile bag with cold water is used because it can be shaped according to the contour of the abdomen and 


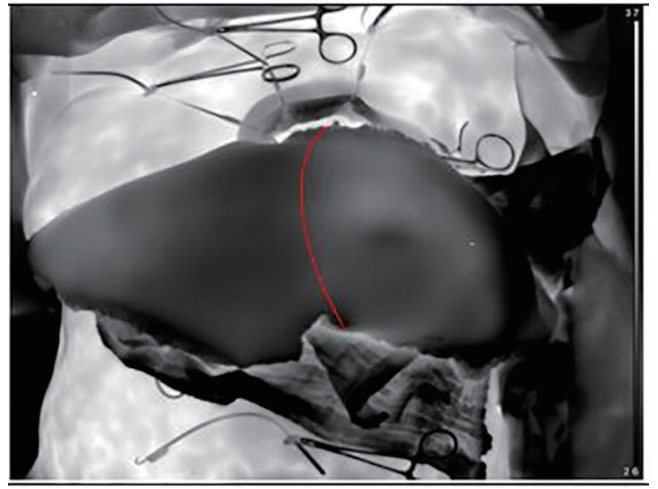

Figure 6 Flap anastomosed in steady state. The red line marks the edge of area with good perfusion.

due to its ability to evenly cool the whole area of interest. Moreover, the use of the bag with water is possible during all moments of the surgical procedure. The choice for a 3 -minute cooling period instead of 10 minutes cooling prevents potential tissue damage. A prolonged exposure of the abdomen to cold temperatures may cause damage to the subdermal plexus and diminish the visibility of hotspots $(17,19)$.

Positioning of the camera is very variable in literature. The optimal position for the IR-camera is perpendicular to the abdominal area. In this position the camera can capture the clearest view of the area of interest. By using a fixed position of the camera in the perpendicular position, the images will not be distorted and automated image analysis will be possible. In other set-ups the camera was a handheld version or the abdomen was framed under an angle (20).

\section{Conclusions}

Multiple studies show that DIRT is a valuable tool during breast reconstruction with free DIEP flaps: it is a safe, lowcost, and quick imaging tool that provides information on location of the perforators, blood flow, and functional characteristics of the vessels.

The measurement set-up for DIEP flap DIRT measurements has a large impact on the results. In this study, a new measurement set-up is proposed that can be applied in the pre-, peri-, and postoperative period of the reconstruction and provides reproducible results. In the future, continuous thermography may be used to detect early venous or arterial problems. Although DIRT is a promising technique, larger studies are needed to provide more conclusive results.

\section{Acknowledgments}

None.

\section{Footnote}

Conflicts of Interest: The authors have no conflicts of interest to declare.

Ethical Statement: The authors are accountable for all aspects of the work in ensuring that questions related to the accuracy or integrity of any part of the work are appropriately investigated and resolved. Ethical approval has been obtained from ethics committee.

\section{References}

1. Blondeel PN. One hundred free DIEP flap breast reconstructions: a personal experience. Br J Plast Surg 1999;52:104-11.

2. Thiessen FEF, Tjalma WAA, Tondu T. Breast reconstruction after breast conservation therapy for breast cancer. Eur J Obstet Gynecol Reprod Biol 2018;230:233-8.

3. Tondu T, Tjalma WAA, Thiessen FEF. Breast reconstruction after mastectomy. Eur J Obstet Gynecol Reprod Biol 2018;230:228-32.

4. Mohan AT, Saint-Cyr M. Advances in imaging technologies for planning breast reconstruction. Gland Surg 2016;5:242-54.

5. Nahabedian MY. Overview of perforator imaging and flap perfusion technologies. Clin Plast Surg 2011;38:165-74.

6. de Weerd L, Mercer JB, Weum S. Dynamic infrared thermography. Clin Plast Surg 2011;38:277-92.

7. de Weerd L, Mercer JB, Setsa LB. Intraoperative dynamic infrared thermography and free-flap surgery. Ann Plast Surg 2006;57:279-84.

8. de Weerd L, Miland AO, Mercer JB. Perfusion dynamics of free DIEP and SIEA flaps during the first postoperative week monitored with dynamic infrared thermography. Ann Plast Surg 2009;62:42-7.

9. de Weerd L, Weum S, Mercer JB. The value of dynamic infrared thermography (DIRT) in perforatorselection and planning of free DIEP flaps. Ann Plast Surg 2009;63:274-9.

10. Weum S, Mercer JB, de Weerd L. Evaluation of dynamic 
infrared thermography as an alternative to CT angiography for perforator mapping in breast reconstruction: a clinical study. BMC Med Imaging 2016;16:43.

11. Tenorio X, Mahajan AL, Elias B, et al. Locating perforator vessels by dynamic infrared imaging and flow Doppler with no thermal cold challenge. Ann Plast Surg 2011;67:143-6.

12. Weum S, Lott A, de Weerd L. Detection of Perforators Using Smartphone Thermal Imaging. Plast Reconstr Surg 2016;138:938e-940e.

13. Whitaker IS, Lie KH, Rozen WM, et al. Dynamic infrared thermography for the preoperative planning of microsurgical breast reconstruction: a comparison with CTA. J Plast Reconstr Aesthet Surg 2012;65:130-2.

14. John HE, Niumsawatt V, Rozen WM, et al. Clinical applications of dynamic infrared thermography in plastic surgery: a systematic review. Gland Surg 2016;5:122-32.

15. Thiessen FEF, Tondu T, Cloostermans B, et al. Dynamic InfraRed Thermography (DIRT) in DIEP-flap breast reconstruction: A review of the literature. Eur J Obstet Gynecol Reprod Biol 2019;242:47-55.

16. Li K, Zhang Z, Nicoli F, et al. Application of Indocyanine Green in Flap Surgery: A Systematic Review. J Reconstr Microsurg 2018;34:77-86.

17. de Weerd L, Weum S, Mercer JB. Dynamic Infrared Thermography (DIRT) in the preoperative, intraoperative and postoperative phase of DIEP flap surgery. J Plast Reconstr Aesthet Surg 2012;65:694-5; author reply 5-6.

Cite this article as: Thiessen FEF, Tondu T, Vermeersch N, Cloostermans B, Lundahl R, Ribbens B, Berzenji L, Verhoeven V, Hubens G, Steenackers G, Tjalma WAA. Dynamic infrared thermography (DIRT) in Deep Inferior Epigastric Perforator (DIEP) flap breast reconstruction: standardization of the measurement set-up. Gland Surg 2019;8(6):799-805. doi: $10.21037 /$ gs.2019.12.09
18. Kolacz S, Moderhak M, Jankau J. New perspective on the in vivo use of cold stress dynamic thermography in integumental reconstruction with the use of skin-muscle flaps. J Surg Res 2017;212:68-76.

19. Zetterman E, Salmi A, Suominen S, et al. Effect of cooling and warming on thermographic imaging of the perforating vessels of the abdomen. Eur J Plast Surg 1999;22:58-61.

20. Walle L, Fansa H, Frerichs O. Smartphone-based thermography for perforator localisation in microvascular breast reconstruction. Handchir Mikrochir Plast Chir 2018;50:111-7.

21. Vollmer M, Möllmann KP. Infrared thermal imaging: fundamentals, research and applications. Weinheim: Wiley-VCH, 2010:593.

22. Kalra S, Dancey A, Waters R. Intraoperative selection of dominant perforator vessel in DIEP free flaps based on perfusion strength using digital infrared thermography - a pilot study. J Plast Reconstr Aesthet Surg 2007;60:1365-8.

23. Lohman RF, Ozturk CN, Ozturk C, et al. An Analysis of Current Techniques Used for Intraoperative Flap Evaluation. Ann Plast Surg 2015;75:679-85.

24. de Weerd L, Weum S, Mercer JB. Detection of perforators using thermal imaging. Plast Reconstr Surg 2014;134:850e-1e.

25. de Weerd L, Weum S, Mercer JB. Locating perforator vessels by dynamic infrared imaging and flow Doppler with no thermal cold challenge. Ann Plast Surg 2014;72:261. 